\title{
Exact renormalization group equations and the field theoretical approach to critical phenomena
}

\author{
C. Bagnuls* and C. Bervillier ${ }^{\dagger}$ \\ C. E. Saclay, F91191 Gif-sur-Yvette Cedex, France
}

February 1, 2008

\begin{abstract}
After a brief presentation of the exact renormalization group equation, we illustrate how the field theoretical (perturbative) approach to critical phenomena takes place in the more general Wilson (nonperturbative) approach. Notions such as the continuum limit and the renormalizability and the presence of singularities in the perturbative series are discussed.
\end{abstract}

This paper has two parts. In the first part we restrict ourselves to the presentation of some selected issues taken from a recent review on the exact renormalization group (RG) equation (ERGE) in the pure scalar case. [1] In the second part we illustrate the Wilson continuum limit of field theory (or, equivalently, what may be understood as the nonperturbative renormalizability). More generally we would like to indicate how the historical first version of the RG, based on a perturbative approach, takes place in the more general nonperturbative framework developed by Wilson. The illustration will be done in the light of actual RG trajectories obtained from a numerical study of the ERGE in the local potential approximation. [2]

\section{Selected issues}

\subsection{What does "exact" mean?}

The word "exact" means: a continuous (i.e. not discrete) realization of the Wilson RG transformation of the action in which no approximation is made and no expansion is involved with respect to some small parameter. We are here only interested in the differential form of the ERGE. One could think that the word exact is not adapted to the Wilson renormalization because, compared to the standard version of renormalization', it involves a finite cutoff and it is only a semi-group. Nevertheless the historical first version is entirely contained in the Wilson theory (see part 2).

\footnotetext{
*Service de Physique de l'Etat Condensé e-mail: bagnuls@spec.saclay.cea.fr

†Service de Physique Théorique e-mail: bervil@spht.saclay.cea.fr

${ }^{1}$ Which originates from perturbation theory.
} 
Vocabulary: With a view to simplify the expression, in the following, the word "action" will systematically replace the customary expression "Wilson's effective action" while the expression "effective action" will be systematically used instead of "Legendre effective action" or "average effective action".

\subsection{Scheme dependence}

In addition to its complexity [an integro-differential equation, see eq. (1)], there is not a unique form of the ERGE. Each form of the equation is characterized by the way the momentum cutoff $\Lambda_{0}$ is introduced. One says that the ERGE is scheme dependent?, but its various forms embody a unique physical content in the sense that they all preserve the same physics at large distances and, via the recourse to a process of limit, yield the same physics at small distances (continuum limits). There exist some studies on the scheme "independence". [3]

Because a sharp boundary in momentum space introduces non-local interactions in position space, [4] the first version of the ERGE has been presented as far back as 1970 [5] with a smooth cutoff introduced via an "incomplete" integration in which large momenta are more completely integrated than small momental. The Wilson version of the ERGE reads: [4]

$$
\dot{S}=\mathcal{G}_{d i l} S+\int_{p}\left(c+2 p^{2}\right)\left(\frac{\delta^{2} S}{\delta \phi_{p} \delta \phi_{-p}}-\frac{\delta S}{\delta \phi_{p}} \frac{\delta S}{\delta \phi_{-p}}+\phi_{p} \frac{\delta S}{\delta \phi_{p}}\right)
$$

in which $S[\phi]$ is the most general action that one may imagine for a scalar field, $\dot{S}=\frac{d S}{d t}$ (with $t=-\ln \frac{\Lambda}{\Lambda_{0}}$ ). The second term in (1) corresponds to the reduction of the degrees of freedom associated with the integration of the high frequency modes of the field and $\mathcal{G}_{\text {dil }} S$ corresponds to the rescaling step of the RG transformation:

$$
\mathcal{G}_{d i l} S=-\left(\int_{p} \phi_{p} \mathbf{p} \cdot \partial_{p} \frac{\delta}{\delta \phi_{p}}+d_{\phi} \int_{p} \phi_{p} \frac{\delta}{\delta \phi_{p}}\right) S
$$

in which $d_{\phi}$ is the classical dimension of the field :

$$
\phi_{s p}=s^{d_{\phi}-d} \phi_{p}
$$

The function $c(t)$ must be adjusted in such a way as to obtain a useful fixed point. [4, 8]

\footnotetext{
${ }^{2}$ In perturbative RG, this kind of dependence is known as the regularization-scheme dependence or "scheme dependence" in short. This should not be confused with the renormalization scheme dependence, a notion associated with the continuum limit which embodies an arbitrariness (see part 2).

${ }^{3}$ An other equation has been derived by Wegner and Houghton [6] for a sharp cutoff and a third one by Polchinski [7] for field theoretical purposes.

${ }^{4}$ Generically speaking: compatible with the symmetry properties of the problem studied.

${ }^{5}$ In principle $d_{\phi}=\frac{d-2}{2}$, but because of the necessity of adjusting the parameter $c$ to get a useful fixed point - where the anomalous dimension $\eta$ of the field may be non-zero- it is customary to let $d_{\phi}$ adjustable instead of $c$, in which case one writes $d_{\phi}=\frac{d-2+\eta}{2}$ with $\eta$ an adjustable constant (near a fixed point).
} 


\subsection{Reparametrization invariance}

This adjustment is a consequence of the independence [8] of the RG transformation on the overall normalization of $\phi$ which is also called "reparametrization invariance". [9] This invariance occurs when the RG transformation is such that the transformed field is related to the original (unintegrated) field by a constant factor as it is presently the case. As consequences:

- a line of equivalent fixed points exists which is parametrized by the normalization of the field,

- a field-rescaling parameter (it is often $\eta$ with $d_{\phi}=\frac{1}{2}(d-2+\eta)$, see footnote 5) takes on a specific value.

- A redundant operator, associated to the change of normalization:

$$
\mathcal{O}_{1}=\int_{q}\left[\frac{\delta^{2} S}{\delta \phi_{q} \delta \phi_{-q}}-\frac{\delta S}{\delta \phi_{q}} \frac{\delta S}{\delta \phi_{-q}}+\phi_{q} \frac{\delta S}{\delta \phi_{q}}\right]
$$

has the eigenvalue [10] $\lambda_{1}=0$ and is absolutely marginall].

\subsection{Effective action}

One may also consider an ERGE for the effective action $\rceil[\Phi]$. It essentially allows to circumvent the singularities induced by the hard cutoff and transforms the UV cutoff into an IR cutoff.

We consider the action with an "additive" IR cutoff $\Lambda$ such that: [12, 9]

$$
S_{\Lambda}[\phi] \equiv \frac{1}{2} \int_{p} \phi_{p} \phi_{-p} C^{-1}(p, \Lambda)+S_{\Lambda_{0}}[\phi]
$$

in which $C(p, \Lambda)$ is an additive infrared cutoff function which is small for $p<\Lambda$ and $p^{2} C(p, \Lambda)$ should be large for $p>\Lambda$.

The ERGE may then be written as follows: [13

$$
\dot{\Gamma}=\mathcal{G}_{d i l} \Gamma+\frac{1}{2} \operatorname{tr} \tilde{\partial}_{t} \ln \left(C^{-1}+\frac{\delta^{2} \Gamma}{\delta \Phi \delta \Phi}\right)
$$

in which $\tilde{\partial}_{t} \equiv-\Lambda \frac{\partial}{\partial \Lambda}$ acts only on $C$ and not on $\Gamma$, i.e. $\tilde{\partial}_{t}=\left(\partial C^{-1} / \partial t\right)\left(\partial / \partial C^{-1}\right)$.

A field theorist's self-consistent approach: There is an efficient short cut for obtaining the ERGE satisfied by the effective action. It is based on the observation that (5) may be obtained from the one loop (unregularized, thus formal) expression of the effective action, which reads (up to a field independent term within the logarithm):

$$
\Gamma[\Phi]=S[\Phi]+\frac{1}{2} \operatorname{tr} \ln \left(\left.\frac{\delta^{2} S}{\delta \phi \delta \phi}\right|_{\phi=\Phi}\right)+\text { higherloop - order }
$$

by using the following practical rules:

\footnotetext{
${ }^{6}$ This is an exception due to the invariance otherwise, in general, a redundant operator does not have a well defined eigenvalue. 11

${ }^{7}$ The effective action is the generating functional of the one-particle irreducible vertex functions.
} 
1. add the infrared cutoff function $C(p, \Lambda)$ of eq. (幽 within the action $S$, eq. (6) then becomes:

$$
\Gamma[\Phi]=\frac{1}{2} \int_{p} \Phi_{p} \Phi_{-p} C^{-1}(p, \Lambda)+S[\Phi]+\left.\frac{1}{2} \operatorname{tr} \ln \left(C^{-1}+\frac{\delta^{2} S}{\delta \phi \delta \phi}\right)\right|_{\phi=\Phi}+\cdots
$$

2. redefine $\tilde{\Gamma}[\Phi]=\Gamma[\Phi]-\frac{1}{2} \int_{p} \Phi_{p} \Phi_{-p} C^{-1}(p, \Lambda)$, then:

$$
\tilde{\Gamma}[\Phi]=S[\Phi]+\left.\frac{1}{2} \operatorname{tr} \ln \left(C^{-1}+\frac{\delta^{2} S}{\delta \phi \delta \phi}\right)\right|_{\phi=\Phi}+\cdots
$$

3. perform the derivative with respect to $\Lambda$, (only the cutoff function is concerned) and forget about the higher loop contributions:

$$
\partial_{t} \tilde{\Gamma}=\frac{1}{2} \operatorname{tr}\left[\frac{1}{C} \Lambda \frac{\partial C}{\partial \Lambda} \cdot\left(1+C \cdot \frac{\delta^{2} S[\Phi]}{\delta \Phi \delta \Phi}\right)^{-1}\right]
$$

4. replace ${ }^{8}$ the action $S$, in the right hand side of the latter equation, by the effective action $\Gamma$ to get (5), the dilatation part $\mathcal{G}_{d i l} \tilde{\Gamma}$ being obtained from usual (engineering) dimensional considerations?.

It is noteworthy that the above rules have been heuristically first used [14 to obtain the local potential approximation of the ERGE for the effective action. However the main interest of the above considerations is that they allow introducing the (infra-red) cutoff function independently of $S$, via the so-called "proper time" (or "heat kernel" or "operator") regularization. [15] This kind of regularization is introduced at the level of eq. (6) via the general identity:

$$
\operatorname{tr} \ln \left(\frac{A}{B}\right)=-\int_{0}^{\infty} \frac{d s}{s} \operatorname{tr}\left(e^{-s A}-e^{-s B}\right)
$$

Forgetting again about the field-independent part (and, momentaneously, about the ultra-violet regularization needed for $s \rightarrow 0$ ) one introduces an infrared cutoff function $F_{\Lambda}(s)$ within the proper time integral representation of the logarithm of $A=\left.\frac{\delta^{2} S}{\delta \phi \delta \phi}\right|_{\phi=\Phi}$ :

$$
\frac{1}{2} \operatorname{tr} \ln A \longrightarrow-\frac{1}{2} \int_{0}^{\infty} \frac{d s}{s} F_{\Lambda}(s) \operatorname{tr} e^{-s A}
$$

The function $F_{\Lambda}(s)$ must tend to zero sufficiently rapidly for large values of $s$ in order to suppress the small momentum modes and should be equal to 1 for $\Lambda=0$.

Then following the rules $3-4$ above applied on $\Gamma$ (i.e., not on $\tilde{\Gamma}$ ), one obtains a new kind of ERGET for the effective action: 16

$$
\partial_{t} \Gamma=-\frac{1}{2} \int_{0}^{\infty} \frac{d s}{s} \Lambda \frac{\partial F_{\Lambda}(s)}{\partial \Lambda} \exp \left[-s \frac{\delta^{2} \Gamma}{\delta \Phi \delta \Phi}\right]
$$

There are apparently two advantages of using this kind of ERGE:

\footnotetext{
${ }^{8}$ This step is often referred to as the "renormalization group improvement" of the one loop effective action.

${ }^{9}$ But do not forget to introduce the anomalous dimension $\eta$ in order to get an eventual nontrivial fixed point.

${ }^{10}$ Notice that, because one performs a derivative with respect to $\Lambda$, the essential contribution to $\partial_{t} \Gamma$ comes from the integration over a small range of values of $s$ (corresponding to the rapid decreasing of $F_{\Lambda}(s)$ ), hence an ultraviolet regularization is not needed provided that the resulting RG equation be finite.
} 
- the regularization preserves the symmetry of the action, 17

- the derivative expansion is slightly easier to perform than in the conventional approach and one may preserve the reparametrization invariance. [16]

Bonano and Zappalà [16] have considered the next-to-leading order of the derivative expansion of (17) (while in a previous work [18] only a pseudo derivative expansion, in which the wave-function renormalization function $Z(\phi, t)$ is field-independent, was used). They have chosen $F_{\Lambda}(s)$ in such a way that the integro-differential character of the ERGE disappears and they have tested the preservation of the reparametrization invariance. Moreover a scheme dependence parameter, related to the cutoff width, is at hand in this framework which, presumably, will allow someone to look at the best possible convergence of the derivative expansion when higher orders will be considered.

Another kind of regularization related to this "self-consistent" approach should be mentioned here. It consists in introducing the cutoff function in (6) in-between the momentum integration [expressing the trace] and the logarithm. This procedure has been considered at the level of the local potential approximation. [19] However the ERGE keeps its integro-differential character and the study [19] has then been conducted within a constraining polynomial expansion method.

\subsection{Lowest order of the derivative expansion}

The study of the ERGE requires the use of approximation (and/or truncation) methods such as the derivative expansion. [20] It is a functional power series expansion of the action in powers of momenta so that all powers of the field are included at each level of the approximation.

In the position space it follows:

$$
S[\phi]=\int d^{d} x\left\{V(\phi, t)+\frac{1}{2} Z(\phi, t)\left(\partial_{\mu} \phi\right)^{2}+\cdots\right\}
$$

The first order of the derivative expansion is the local potential approximation in which $Z(\phi, t) \equiv z$ is a constant and $V(\phi, t)$ a simple function which, sometimes, we conveniently represent as a sum of monomials of $\phi$ as, e.g., in the case of the $Z_{2}$ symmetry:

$$
V(\phi, t)=\sum_{k=1}^{\infty} u_{2 k}(t) \phi^{2 k}
$$

In this approximation [21] the ERGE reduces to a differential equation for $V(\phi, t)$ which, with the Wilson version (1), reads:

$$
\dot{V}=V^{\prime \prime}-\left(V^{\prime}\right)^{2}+\left(1-\frac{d}{2}\right) \phi V^{\prime}+d V
$$

In the approximation, the parameter $\eta$ must take on the value 0 .

Interest: The local potential approximation allows to consider all powers of $\phi$ on the same footing. It provides us with an excellent textbook example to illustrate (and to qualitatively investigate) the nonperturbative aspects of the Wilson theory $\square$. The

\footnotetext{
${ }^{11}$ Exact results are accessible within this approximation. Wegner and Houghton [6] have shown that the limit $N \rightarrow \infty$ of their ERGE in the $O(N)$-symmetric case is identical to the limit $N=\infty$ of the local potential approximation. Now the limit $N=\infty$ corresponds to the spherical model which is exactly solvable.
} 
only lacking features are related to phenomena highly correlated to the non local parts neglected in the approximation. For example in two dimensions $(d=2)$, where $\eta=\frac{1}{4}$ is not particularly small, the local potential approximation is unable to display the expected fixed point structure. [22] Otherwise, when $\eta$ is small (especially for $d=4$ and $d=3$ ), one expects the approximation to be qualitatively correct on all aspects of the RG theory. Especially on the number of existing nontrivial fixed points.

Fixed points: The fixed points are solutions of the equation $\dot{V}=0$. This is a non linear second order differential equation. A nontrivial solution is parametrized by two arbitrary constants. By imposing, e.g. $V^{* \prime}(0)=0$ for an even function of $\phi$, we are left with a one-parameter family of (nontrivial) solutions to the differential equation. However, all but a finite number of the solutions in the family are singular at some $\phi_{c}$. By requiring the physical fixed point to be defined for all $\phi$ then the acceptable fixed points (if they exist) may all be found by adjusting one parameter in $V(\phi)$.

Critical exponents: The best way to calculate the critical exponents is to linearize the flow equation in the vicinity of the fixed point and to look at the eigenvalue problem. One obtains a linear second order differential equation. For example with the Wilson version (11), setting

$$
V(\phi, t)=V^{*}+e^{\lambda t} v(\phi)
$$

the eigenvalue equation reads:

$$
v^{\prime \prime}+\left[\left(1-\frac{d}{2}\right) \phi-2 V^{* \prime}\right] v^{\prime}+(d-\lambda) v=0
$$

Again one expects solutions to this equation labelled by two parameters, however by linearity one can choose $v(0)=1$ (arbitrary normalization of the eigenvectors) and by symmetry $v^{\prime}(0)=0$. Thus the solutions are unique, given $\lambda$. Now for large $\phi, v(\phi)$ is generically a superposition of $v_{1} \sim \phi^{2(d-\lambda) /(d+2)}$ and of $v_{2} \sim \exp \left(\frac{d+2}{4} \phi^{2}\right)$. Requiring zero coefficient for the latter restricts the allowed values of $\lambda$ to a discret set". 9]

The reason for which the exponential must be eliminated is precisely the necessity of having a quantized set of eigenvalues. This necessity is related to the notion of renormalizability or, equivalently, of self-similarity which states that the effect of the infinite number of degrees of freedom involved in a field theory are all accounted for by means of a (small) discrete set of renormalized parameters (see part 2). This precise detail is important with respect to the existence of nonpolynomial solutions giving rise to relevant directions for the Gaussian fixed point. 23] This would make the scalar field theory in four dimensions asymptoytically free. It exists recent studies of these new relevant directions, 24] however the set of relevant directions is a continuum and this is not in agreement [25, 11] with the standards of the renormalization theory (see part 2).

Next-to-leading order in the derivative expansion: There, $Z(\phi, t)$ is no longer a constant and a system of two coupled equations for $V$ and $Z$ takes place. We do not write down them here, we simply aim at mentioning the breaking of the reparametrization invariance. This means that instead of having a line of equivalent fixed points generated by the change of normalization of the field, one gets a line of non equivalent fixed points each being associated with a different value of the field-rescaling parameter $\eta$. However a residual effect of the invariance allows to determine an optimal value ${ }^{\mathrm{T}}$ of $\eta$. [8, 20]

\footnotetext{
${ }^{12}$ See also J. Comellas. 3
} 
The derivative expansion generally breaks the reparametrization invariance except with two regularization schemes:

- the sharp cutoff scheme, but the derivative expansion is delicate to define in that case. [26]

- the pure power law cutoff function of the form: [9, 27]

$$
\tilde{C}\left(p^{2}\right)=p^{2 k}
$$

In that case a unique value of $\eta$ is found and also the eigenvalue 0 corresponding to the redundant operator $\mathcal{O}_{1}$ mentioned previously.

Convergence of the derivative expansion: One does not know whether the expansion converges or not. [28] It could be Borel summable however. [29]

Conclusion: Supplementary works on this expansion would be welcome:

- Calculation of higher orders

- Studies on its convergent or divergent nature

- Looking for new kinds of approximations applied to the ERGE (see, e.g. Golner [30]).

To those who would have some doubts about the interest of pursuing those studies, we recommend to look at the rich variety of calculations that may be done within the nonperturbative Wilson's approach for example in the papers by Wetterich and coworkers. [31]

\section{Wilson's continuum limit (or nonperturbative renor- malizability)}

The expression "continuum limit" comes from the consideration of a field theory on a lattice for which one would like to make the lattice spacing $a$ vanish $(a \rightarrow 0)$. In fact, the Wilson theory allows us to consider special kinds of actions which already display the fantastic property of evolving (under the effect of a RG transformation) as if the lattice spacing was actually zero although it is not. These actions are called "improved actions" [32, 33] or "perfect actions" [34] according to the method used to construct them[t].

Actually, the continuum limit of a lattice field theory leads to a nonperturbative equivalent expression of the notion of renormalizability ${ }^{\text {tat }}$. This relies essentially upon the following correspondence:

- in one hand (perturbation theory), the renormalizability states that one may push the initial cutoff $\Lambda_{0}\left(\sim \frac{1}{a}\right)$ to infinity while defining only a finite (usually small) number of renormalized parameters;

\footnotetext{
${ }^{13}$ Respectively perturbatively (improved) and nonperturbatively (perfect).

${ }^{14} \mathrm{~A}$ notion inherited from perturbation theory.
} 
- in the other hand (Wilson's theory), in the same limit $\left(\Lambda_{0} \rightarrow \infty\right)$, the nature of the renormalized parameters and their number are given by the finite set (usually small) of relevant directions of a fixed point.

But, be careful, the expression: "the relevant parameters are the renormalized parameters" is incomplete (and thus incorrect). This is because there are two inseparable aspects in the definition of a renormalized parameter:

1. its nature: e.g., is it a $\phi^{4}$-like or a $\phi^{6}$-like coupling or something else?

2. the RG flow which runs along a renormalized trajectory (RT) emerging from the fixed point. This flow is usually expressed via a $\beta$-function expressing a momentum dependence of the renormalized coupling (the nature of which is already specified).

A relevant parameter has the same nature as the renormalized parameter but, because the notion of relevance is attached to a linearization of the $R G$ transformation in the vicinity of a fixed point, [11] the flow carried by a relevant parameter runs locally only tangentially to the trajectory of actual interest. Consequently, the second characteristics (the RG flow along the RT) is not correctly expressed by the relevant character of a parameter.

It is the aim of the following considerations to illustrate and discuss these issues.

Discussion of a traditional figure: Since the famous review by Wilson and Kogut, [4 one invariably illustrates the notion of continuum limit with the once unstable fixed point configuration giving rise to a purely massive renormalized field theory. 35] But it is not the best candidate $\mathrm{T}^{\mathrm{T}}$ for an illustration of the most important aspect of the RG in field theory, namely, the momentum-scale dependence of the renormalized parameters (i.e., in the continuum limit). [2] Let us first discuss briefly this traditional figure (see figs. 11 and 2).

In the Wilson space $\mathcal{W}$ (of infinite dimension $D_{\mathcal{W}}$ ) of the action-parameters, there is the critical submanifold $\mathcal{W}_{c}$ of dimension $D_{\mathcal{W}}-1$ in which, when $d=3$, lies the Wilson-Fisher fixed point (with only one direction of infrared instability). If we initialize a bare action in $\mathcal{W}_{c}$, then the RG transformation carries the running action toward the fixed point. If the bare action is chosen very close to $\mathcal{W}_{c}$, then the renormalization trajectory passes close to the fixed point and finally goes away from $\mathcal{W}_{c}$ essentially along a limiting trajectory which ideally emerges from $\mathcal{W}_{c}$ at the fixed point. This trajectory is a RT because if we formally associate a point of it to a finite and fixed momentum scale $\Lambda$ then, since an infinite time is required to go out from the fixed point, the initial cutoff $\Lambda_{0}$ (assumed to be associated to the fixed point) appears to be infinite with respect to $\Lambda$. Then it is an obvious matter to show that the relevant parameter (presently a mass) takes on a finite value at this point and that, consequently, a finite (renormalized) mass-parameter exists in the limit $\Lambda_{0} \rightarrow \infty$.

This issue, which specifies the nature of the renormalized parameters in the continuum limit, clearly illustrates the crucial aspect of the renormalizability: namely the small number of the renormalized parameters finally involved

\footnotetext{
${ }^{15}$ In the purely massive case, the mass may be, at the same time, a scale of reference and a scaledependent (renormalized) parameter. This fact obscures the (momentum) scale dependence.

${ }^{16}$ The continuum of relevant directions of the Gaussian fixed point found in four dimensions with nonpolynomial forms of the action [23] contradicts [25, 11 this aspect of the RGT (the fixed point then possesses an infinite number of unstable directions).
} 
The second issue (the specification of the RG flow) is the main content of the RGT. The RG flow in the continuum is precisely (obviously) the flow which runs along the RT. Ideally, to get it in the exact RGT, we could initialize the action at a point lying right on the RT (perfect action). Although the corresponding cutoff is $\Lambda_{0}$, the perfect action is "renormalized" (the continuum limit is reached) in the sense that the subsequent evolution under a $R G$ transformation is unique (for a given fixed point).

Hence, the continuum limit could be obtained by imposing a perfect action as initial condition to the ERGE. But, since the RT is entirely plunged in a space of infinite dimension, an infinite number of action-parameters must be specified. Because this cannot be realized in practice, one has recourse either to a truncation (approximate perfect action) or to a limiting process consisting in a fine tuning of the bare action somewheret] close to $\mathcal{W}_{c}$.

In perturbation theory an initial complicated action is never considered explicitly. On the contrary, one systematically insists on the fact that the renormalized action remains simple (i.e., does not involve an infinite number of parameters). Hence, the latter mentioned facet of the Wilson theory seems to contradict the notion of renormalizability which we were particularly attached to.

Actually, in the expression of the RG flow in the "continuum", we distinguish two things:

a) the RG flow itself characterized by its instantaneous speed. It may be expressed with a small number of flowing parameters ${ }^{18}$ the nature of which is that of the relevant parameters at the fixed point. The scale dependence then expresses under a differential form ( $\beta$-functions). It is that aspect of the renormalization of field theory which is privilegiated in the perturbative approach.

b) the initialization of the $\mathrm{RG}$ flow. It requires the specification of an infinite number of conditions on the action. This aspect of the renormalization of field theory is nonperturbative by nature. We refer to it as the functional form of the scale dependence in the continuum limit.

A legitimate question then arises. Since in constructing perturbatively the renormalized theory we never refer explicitly to a fixed point $t^{\text {TI }}$ - and a fortiori to the notion of relevance -, and also we never consider any perfect action [20, how the right RG flow has been chosen allowing us to get "the best" estimates of the 3- $d$ critical exponents even though these quantities are highly nonperturbative in nature?

The answer is: by picking out a particular RG flow which turns out to be the slowestet in $\mathcal{W}$. Let us illustrate this with the massless $\phi_{3}^{4}$-theory.

The 3- $d$ massless field theory: A massless theory is defined in the critical submanifold $\mathcal{W}_{c}$. Because the renormalization of field theory gives rise to a scale dependence, the massless theory of interest is not defined at the fixed point. Of course, a fixed point theory is scale invariant and could correspond to a theory without mass (since a mass would break the scale invariance). But, despite this marvellous and fascinating property

\footnotetext{
${ }^{17}$ Only one action-parameter (e.g., a bare mass) has to be fine tuned in the purely massive case.

${ }^{18}$ Hence the notion of self similarity: the system looks like the same at any momentum scale of reference.

${ }^{19}$ Except implicitly to the Gaussian fixed point.

${ }^{20}$ Except in the Symanzik improvement program [32 for lattice field theory.

${ }^{21}$ Asymptotically close to the Gaussian fixed point, this corresponds to having chosen the relevant flow.
} 
of scale invariance, at a fixed point everything is fixed, there is no scale dependence and in fact, there is nothing interesting to describe.

The 3- $d$ massless theory which we are interested in is scale dependent and it is defined by reference to a $\mathrm{RG}$ flow running in $\mathcal{W}_{c}$ and emerging from the Gaussian fixed point 22 .

Fig. 3 displays the same fixed point configuration as fig. 2 but with a larger view which includes the Gaussian fixed point. We can see a RT which emerges from the Gaussian FP. It is the RT on which is defined the continuum limit of the massless field theory for $d=3$.

Fig. 4 shows exclusively the (projected) RG flows in the critical submanifold $\mathcal{W}_{c}$. We observe critical flows reaching the Wilson-Fisher fixed point. To get these critical trajectories we must adjust, for each of them, one parameter of the initial action (critical temperature).

The RT for the massless field theory interpolates between the Gaussian and the WilsonFisher fixed points. It is an (infrared) attractive one-dimensional submanifold. It is like a large river into which the ordinary Wilson flows run. It corresponds to the slowest flow in the Wilson space. [2]

Fig. 1 is static: it only shows RG trajectories. Fig. 5 is a dynamic picture of the flows showing the evolution of the $\phi^{4}$-coupling $u_{4}(t)$ along the critical (massless) RT. We obtain this way an image of the functional forms of the scale dependence in the continuum which differ only by one initial condition. If we require that each flow reaches the same value of $u_{4}$ (the same point in $\mathcal{W}$ ) at the same time, then we obtain an unique evolution except for some marginal "finite cutoff" effects corresponding to those trajectories which were initialized far from the Gaussian fixed point.

Expressed under the differential form, we get a unique $\beta$-function: $\beta\left(u_{4}\right)=\Lambda d u_{4} / d \Lambda$ (see fig. 6). The $\beta$-function so obtained expresses the uniqueness of the slowest flow along the RT which interpolates between the Gaussian and Wilson-Fisher fixed point (in the critical submanifilod $\mathcal{W}_{c}$ ). It is precisely that flow which has been picked out by the perturbative approach while applying the subtraction procedure required to make finite, order by order, the perturbative series of the $\phi_{4}^{4}$-theory in the limit $\Lambda_{0} \rightarrow \infty$. So defined as carrier of the differential RG flow running along the RT, $u_{4}(t)$ is like the usual renormalized $\phi^{4}$-coupling of perturbation theory ${ }^{23}$.

Once the ideal flow has been selected near the Gaussian fixed point, it remains to calculate the perturbative series and to Borel-resum them up to the Wilson-Fisher fixed point in order to estimate the critical exponents. [36]

Now this is not the whole story, because, when $d=4$, there are ultraviolet renormalon singularities in the perturbative series which prevent them from being Borel summable. [37]

In four dimensions, the Wilson-Fisher fixed point disappears and all the critical RG flows run toward the Gaussian fixed point (see fig. 7).

We see that, if the ideal slowest flow is underlying (all the trajectories are attracted to an infrared stable submanifold of dimension one), it cannot be exactly reached by the

\footnotetext{
${ }^{22}$ One usually says that the theory is defined "at" the Gaussian fixed point but it is not an actual fixed-point theory defined at the fixed point.

${ }^{23}$ Notice the freedom in the choice of the renormalized parameter (arbitrariness of the renormalization scheme). Indeed, it is only an intermediary in the expression of the essential RG flow of the action running along the RT. We could have chosen a parameter different from $u_{4}$ provided that asymptotically close to the Gaussian fixed point it behaves like the $\phi^{4}$-coupling which coincides with the relevant direction at this fixed point as shown in fig. 1 .
} 
$\mathrm{RG}$ flows because there is no once-unstable fixed point in $\mathcal{W}_{c}$ which could canalize all the degrees of freedom of the theory along an unique ideal (perfect) trajectory yielding a complete momentum scale dependence in the range ]0, $\infty[$. Consequently, the Wilson trajectories approach the ideal trajectory in the infrared regime but never reach it exactly and there remain ambiguities between the actual and the ideal trajectories especially in the ultraviolet regime (i.e., at least for the momentum-scale of reference larger to some finite $\Lambda_{\max }$ ). The only possibility of reaching a pseudo "continuum limit" (i.e., of reaching the ideal, but incomplete, slowest RG flow) would be to explicitly write down the perfect action which associates a point of the underlying ideal trajectory to $\Lambda$ (initial condition on the functional form of the momentum scale dependence). However an infinity of conditions are required and moreover the range of variation of the momentum-scale would remain finite (] $\left.\left.0, \Lambda_{\max }\right]\right)$ due to the lack of ultraviolet stable fixed point. Ambiguities are then induced in the process of defining of the initial (perfect) action in the ultraviolet regime.

Ambiguities of the same nature as the preceding ones exist also in perturbation theory, they are due to ultraviolet renormalons. [37] It has been shown by Parisi [38 and then by Bergère and David [39] that the renormalons could be removed from the theory by considering an infinite number of composite operators and this would amount to reintroducing a "finite cutoff". [39] In fact, this removal is nothing but the construction of the perfect action (initialization of the functional form of the momentum-scale dependence). Indeed it has been shown that even in the absence of an ultraviolet stable fixed point, the perturbative series of the $\phi_{4}^{4}$ field theory are Borel summable [40] provided that the RG flow be initialized somewhere and identifiable at any smaller cutoff scale ${ }^{20}$ (infra-red $\phi_{4}^{4}$ model $[20$.

In the continuum limit, the problem of dealing with the infinite number of degrees of freedom is completely involved in the initial condition of the ideal scale dependence provided we have first picked out the slowest flow. If the ultraviolet stable fixed point is lacking then the initial condition is obliged and introduces ambiguities. Otherwise, all the degrees of freedom are exactly canalized in the relevant direction leaving no room for any ambiguity (the obliged initial condition takes place "at" the fixed point).

Now the following question arises: Why the $\beta$-function perturbatively determined would display renormalon singularities since it expresses a simple flow describable by a single variable while the renormalons are related to the specification of an obliged initial condition (involving explicitly the infinity of degrees of freedom of the theory)?

The answer is that in the definition of the $\beta$-function, there are two aspects:

1. the slowest flow itself

2. the running variable chosen to express this flow

If we choose the renormalized coupling via a renormalization-point condition then the question of dealing explicitly with the infinity of degrees of freedom (determination of a

\footnotetext{
${ }^{24}$ Notice that when a fixed point exists only a few numbers of action-parameters have to be fine tuned "at" the fixed point to reach the (unique) continuum limit. However an infinity of fine tunings are allowed giving rise to a family of functional forms of the momentum-scale dependence (by varying the initial condition).

${ }^{25}$ That is to say, provided that a definite RG flow is chosen once and for all.

${ }^{26}$ The infra-red- $\phi_{4}^{4}$-model is a massless theory with an ultra-violet cutoff. Its renormalization flows have been also rigorously studied. [41]
} 
vertex function) is raised and there is a gap between the ideal flow and the variable chosen. Consequently, there are renormalon singularities in the $\beta$-function in four dimensions.

However if we choose a renormalization scheme in such a way as to exclusively refer to the ideal slowest flow without making any reference to any vertex function, then the $\beta$-function in four dimensions would have no renormalon singularities at all and it would be Borel summable. Such renormalization schemes exist, they are called minimal subtraction schemes. In such schemes, one only subtracts what is necessary to make the theory finite when $d=4$, the renormalized coupling is not a vertex function, it is some intermediate parameter not particularly defined in other respects and the momentum scale of reference is purely artificial. In doing so one has exclusively referred to the ideal slowest flow. Hence the $\beta$-function determined in a minimal subtraction scheme could be Borel summable in four dimensions while the whole field theory remains trivial due to the renormalons singularities. This proposal agrees with the fact "that it is possible to define renormalization schemes such that the renormalization group functions $[\beta(u), \eta(u), \cdots]$ do not have ultra-violet renormalons". [39]

Using the same process as above, we could have also discussed [2] the singularities at the fixed point in the $\beta$-function in three dimensions (first envisaged by Nickel [42] and also more recently studied [43]) and the RG trajectories in the sector $u_{4}<0$. [44]

\section{Figure captions}

1. The traditional once unstable fixed point configuration, see also fig. 2 .

2. This figure, similar to fig. 1, reproduces the projection onto a plane of actual RG trajectories obtained from a nonperturbative study in three dimensions of the ERGE in the local potential approximation. [2] It illustrates the simplest nonperturbative continuum limit in three dimensions: Approach to the purely massive "renormalized trajectory" $T_{0}$ (dot-dashed curve) by RG trajectories initialized at $u_{4}(0)=3$ and $u_{n}(0)=0$ for $n>4$ and $\left(u_{2}(0)-u_{2}^{c}\right) \rightarrow 0^{+}$(open circles) in which $u_{2}^{c}$ corresponds to the adjustment of the initial action in order to approach the Wilson-Fisher fixed point (black full circle, W.-F.) as in figure $₫$. The trajectories drawn correspond to $\log \left(u_{2}(0)-u_{2}^{c}\right)=-1,-2,-3,-4,-5,-6$. Arrows indicate the infrared direction.

3. Same figure as fig. 2 with a larger view including the Gaussian fixed point. It illustrates the continuum limit of the massive $\phi_{3}^{4}$-theory defined "at" the twiceunstable Gaussian fixed point and involving two renormalized parameters (a mass and a coupling constant). The open circles represent initial actions corresponding to different "fine" tunings "at" the Gaussian fixed point. To get the massless $\phi_{3}^{4}$ theory one must adjust, in addition, one action-parameter (e.g., $u_{2}(0)$ to its critical value $\left.u_{2}^{c}\right)$ in order to make the action running along the ideal trajectory $\left(\mathrm{T}_{1}\right)$ which interpolates between the two fixed points (Gaussian and Wilson-Fisher) indicated by full black circles.

4. Projection onto the plane $\left(u_{4}, u_{6}\right)$ of some critical $R G$ trajectories for $u_{4}(0)>0$ [from a numerical study [2] with $d=3$ ]. Full lines represent trajectories on the critical submanifold $\mathcal{W}_{c}$. The arrows indicate the directions of the $\mathrm{RG}$ flows on the trajectories. The submanifold $\mathrm{T}_{1}$ of one dimension (which the trajectories are 
attracted to) which links the Gaussian fixed point to the Wilson-Fisher fixed point corresponds to the renormalized trajectory on which is defined the continuum limit of the massless field theory in three dimensions. The open circles represent initial simple actions.

5. Functional momentum-scale dependence displayed by $u_{4}(t)$ along definite RG flows in $\mathcal{W}_{c}$ (lefthand figure). Open circles indicate the initial points chosen in $\mathcal{W}_{c}$. Each full curve provides a determination of the functional momentum-scale dependence. In the righthand figure we have artificially translated the "time" scales $t$ (vertical dashed lines) such that each actual Wilson's flow "hits" a given unique value of $u_{4}$ at the same "time". The unique functional flow so obtained illustrates the underlying unique differential flow of fig. 5 .

6. Graphical representation of the differential $\mathrm{RG}$ flows along the submanifold $\mathrm{T}_{1}$ of fig. 1 projected on the $u_{4}$-axis -i.e. $\beta\left(u_{4}\right)=-d u_{4}(t) / d t$. In the infra-red direction, any critical RG flow exponentially approaches the limiting RT $\mathrm{T}_{1}$ along which the flows coincide with the unique differential-momentum-scale-dependence carried by the ultimate Wilson flow emerging from the Gaussian fixed point (origin). The righthand zero corresponds to the Wilson-Fisher infra-red stable fixed-point-value [2] $u_{4}^{*}=3.27039 \cdots$

7. RG trajectories on the critical submanifold $\mathcal{W}_{c}$ when $d=4$ (projection onto the plane $\left.\left(u_{4}, u_{6}\right)\right)$. Open circles represent initial simple actions chosen in $\mathcal{W}_{c}$ (of codimension 1). The two lines which come from the upper side of the figure are $\mathrm{RG}$ trajectories initialized at $u_{4}(0)=20$ and $u_{4}(0)=40$ respectively. The arrows indicate the infrared direction. The trajectories are attracted to a submanifold of dimension one before plunging into the Gaussian fixed point. This is a pseudo renormalized trajectory (it has no well defined beginning) and strictly speaking, the continuum limit does not exist due to the lack of another (nontrivial) fixed point which would allow the scale dependence (of the renormalized parameter along the RT) to be defined in the whole range of scale $] 0, \infty[$. See text for a discussion (from a numerical study [2]).

\section{References}

[1] C. Bagnuls and C. Bervillier, "Exact Renormalization Group Equations. An Introductory Review.", eprint: hep-th/0002034, to appear in Phys. Rep.

[2] C. Bagnuls and C. Bervillier, J. Phys. Stud. 1, 366 (1997); see also Phys. Rev. B41, 402 (1990).

[3] R. D. Ball, P. E. Haagensen, J. I. Latorre and E. Moreno, Phys. Lett. B347, 80 (1995). D. F. Litim, Phys. Lett. B393, 103 (1997); Phys. Lett. B486, 92 (2000). J. Comellas, Nucl. Phys. B509, 662 (1998). J.-I. Sumi, W. Souma, K.-I. Aoki, H. Terao and K. Morikawa, eprint: hep-th/0002231. J. I. Latorre and T. R. Morris, J. High Energy Phys. 0011, 004 (2000).

[4] K. G. Wilson and J. Kogut, Phys. Rep. 12C, 77 (1974). 
[5] K. G. Wilson, in "Irvine Conference (unpublished)" (1970).

[6] F. J. Wegner and A. Houghton, Phys. Rev. A8, 401 (1973).

[7] J. Polchinski, Nucl. Phys. B231, 269 (1984).

[8] T. L. Bell and K. G. Wilson, Phys. Rev. B10, 3935 (1974); ibid. B11, 3431 (1975).

[9] T. R. Morris, Phys. Lett. B329, 241 (1994).

[10] E. K. Riedel, G. R. Golner and K. E. Newman, Ann. Phys. (N.Y.) 161, 178 (1985).

[11] F. J. Wegner, in Phase Transitions and Critical Phenomena Vol. VI, p. 7, Ed. by C. Domb and M.S. Green (Acad. Press, N.-Y., 1976).

[12] C. Wetterich, Phys. Lett. B301, 90 (1993).

[13] D.-U. Jungnickel and C. Wetterich, in "The Exact Renormalization Group", p. 41, Ed. by A. Krasnitz, R. Potting, P. Sá and Y. A. Kubyshin (World Scientific, Publ. Co., Singapore, 1999).

[14] N. Tetradis and C. Wetterich, Nucl. Phys. B398, 659 (1993). C. Wetterich, Z. Phys. C57, 451 (1993). S.-B. Liao and J. Polonyi, Ann. Phys. (N.Y.) 222, 122 (1993); Phys. Rev. D51, 4474 (1995).

[15] R. Floreanini and R. Percacci, Phys. Lett. B356, 205 (1995). S.-B. Liao, Phys. Rev. D53, 2020 (1996).

[16] A. Bonanno and D. Zappalà, eprint: hep-th/0010095, to appear in Phys. Lett. B.

[17] M. Oleszczuk, Z. Phys. C64, 533 (1994).

[18] O. Bohr, B.-J. Schaefer and J. Wambach, eprint: hep-ph/0007098.

[19] S.-B. Liao, C.-Y. Lin and M. Strickland, eprint: hep-th/0010100.

[20] R. J. Myerson, Phys. Rev. B12, 2789 (1975). G. R. Golner, Phys. Rev. B33, 7863 (1986).

[21] J. F. Nicoll, T. S. Chang and H. E. Stanley, Phys. Rev. Lett. 33, 540 (1974); Phys. Rev. A13, 1251 (1976). V. I. Tokar, Phys. Lett. A104, 135 (1984). A. Hasenfratz and P. Hasenfratz, Nucl. Phys. B270 [FS16], 687 (1986).

[22] A. E. Filippov and S. A. Breus, Phys. Lett. A158, 300 (1991). S. A. Breus and A. E. Filippov, Physica A192, 486 (1993). A. E. Filippov, Theor. Math. Phys. 91, 551 (1992). T. R. Morris, Phys. Lett. B345, 139 (1995).

[23] K. Halpern and K. Huang, Phys. Rev. Lett. 74, 3526 (1995); Phys. Rev. D53, 3252 (1996). V. Periwal, Mod. Phys. Lett. A11, 2915 (1996).

[24] K. Halpern, Phys. Rev. D57, 6337 (1998). A. Bonanno, Phys. Rev. D62, 027701 (2000). V. Branchina, eprint: hep-ph/0002013. H. Gies, eprint: hep-th/0009041, to appear in Phys. Rev. D. 
[25] T. R. Morris, Phys. Rev. Lett. 77, 1658 (1996).

[26] T. R. Morris, Nucl. Phys. B458 [FS], 477 (1996).

[27] T. R. Morris, Int. J. Mod. Phys. A9, 2411 (1994).

[28] T. R. Morris and J. F. Tighe, J. High Energy Phys. 08, 007 (1999).

[29] G. V. Dunne, Int. J. Mod. Phys. A12, 1143 (1997). G. V. Dunne and T. M. Hall, Phys. Rev. D60, 065002 (1999).

[30] G. R. Golner, eprint: hep-th/9801124.

[31] J. Berges, N. Tetradis and C. Wetterich, "Non-Perturbative Renormalization Flow in Quantum Field Theory and Statistical Physics", eprint: hep-ph/0005122, to appear in Phys. Rep.

[32] K. Symanzik, in Mathematical Problems in Theoretical Physics, Ed. by R. Schrader et al (Springer, Berlin, 1982); Nucl. Phys. B226, 187 (1983); ibid. B226, 205 (1983).

[33] C. Wieczerkowski, Comm. Math. Phys. 120, 149 (1988).

[34] P. Hasenfratz, Prog. Theor. Phys. Suppl. 131, 189 (1998); in "Confinement, duality, and nonperturbative aspects of QCD", p. 179, Ed. by P. van Baal (Plenum Press, N.-Y., 1998).

[35] K. G. Wilson, Phys. Rev. D6, 419 (1972). G. R. Golner, Phys. Rev. D8, 3393 (1973).

[36] J. C. Le Guillou and J. Zinn-Justin, Phys. Rev. Lett. 39, 95 (1977); Phys. Rev. B21, 3976 (1980). R. Guida and J. Zinn-Justin, J. Phys. A31, 8103 (1998).

[37] D. J. Gross and A. Neveu, Phys. Rev. D10, 3235 (1974).

[38] G. Parisi, Phys. Lett. 76B, 65 (1978); Phys. Rep. 49, 215 (1979).

[39] M. C. Bergère and F. David, Phys. Lett. 135B, 253 (1984).

[40] J. S. Feldman, J. Magnen, V. Rivasseau and R. Sénéor, Comm. Math. Phys. 109, 437 (1987).

[41] K. Gawedski and A. Kupiainen, Phys. Rev. Lett. 54, 92 (1985); Comm. Math. Phys. 99, 197 (1985).

[42] B. G. Nickel, in Phase Transitions Ed. by M. Lévy, J. C. Le Guillou and J. Zinn-Justin (Plenum, New York and London, 1982); Physica A177, 189 (1991).

[43] B. Li, N. Madras and A. D. Sokal, J. Stat. Phys. 80, 661 (1995). A. Pelissetto and E. Vicari, Nucl. Phys. B519, 626 (1998); Nucl. Phys. B (Proc. Suppl.) 73, 775 (1999). P. Calabrese, M. Caselle, A. Celi, A. Pelissetto and E. Vicari, J. Phys. A33, 8155 (2000). M. Caselle, A. Pelissetto and E. Vicari, eprint: hep-th/0010228 (2000).

[44] C. Bagnuls and C. Bervillier, Cond. Matt. Phys. 3, 559 (2000). 


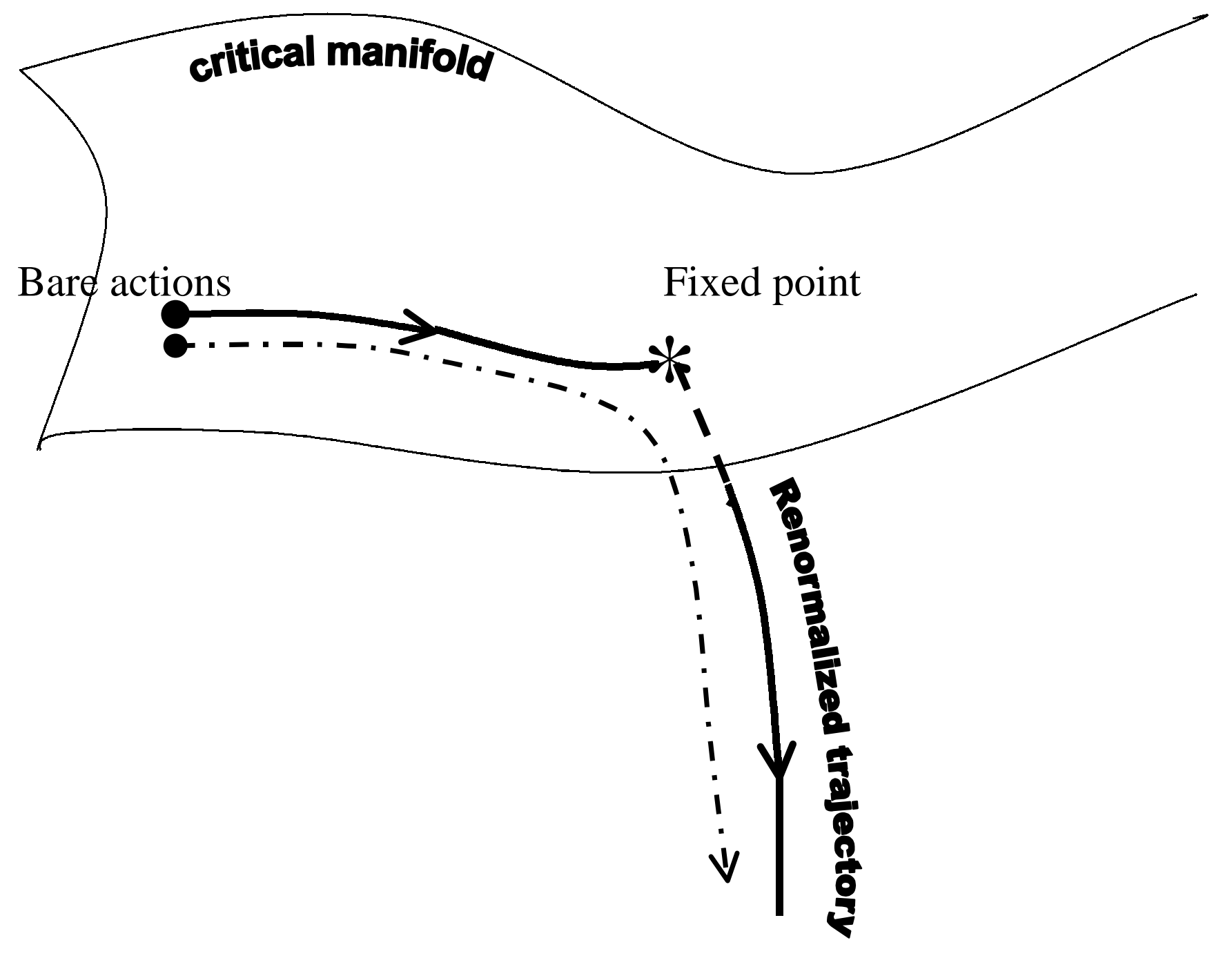




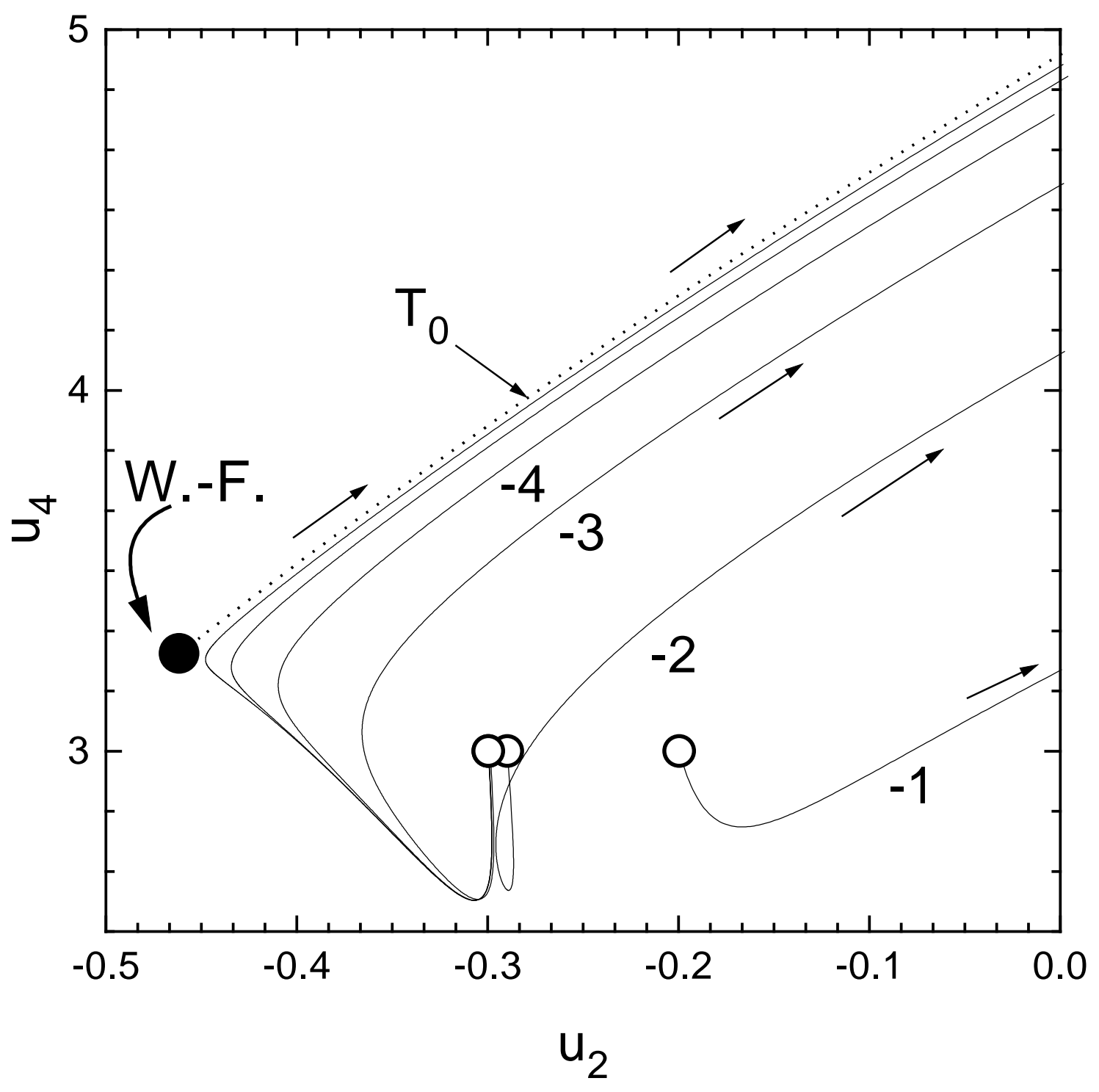




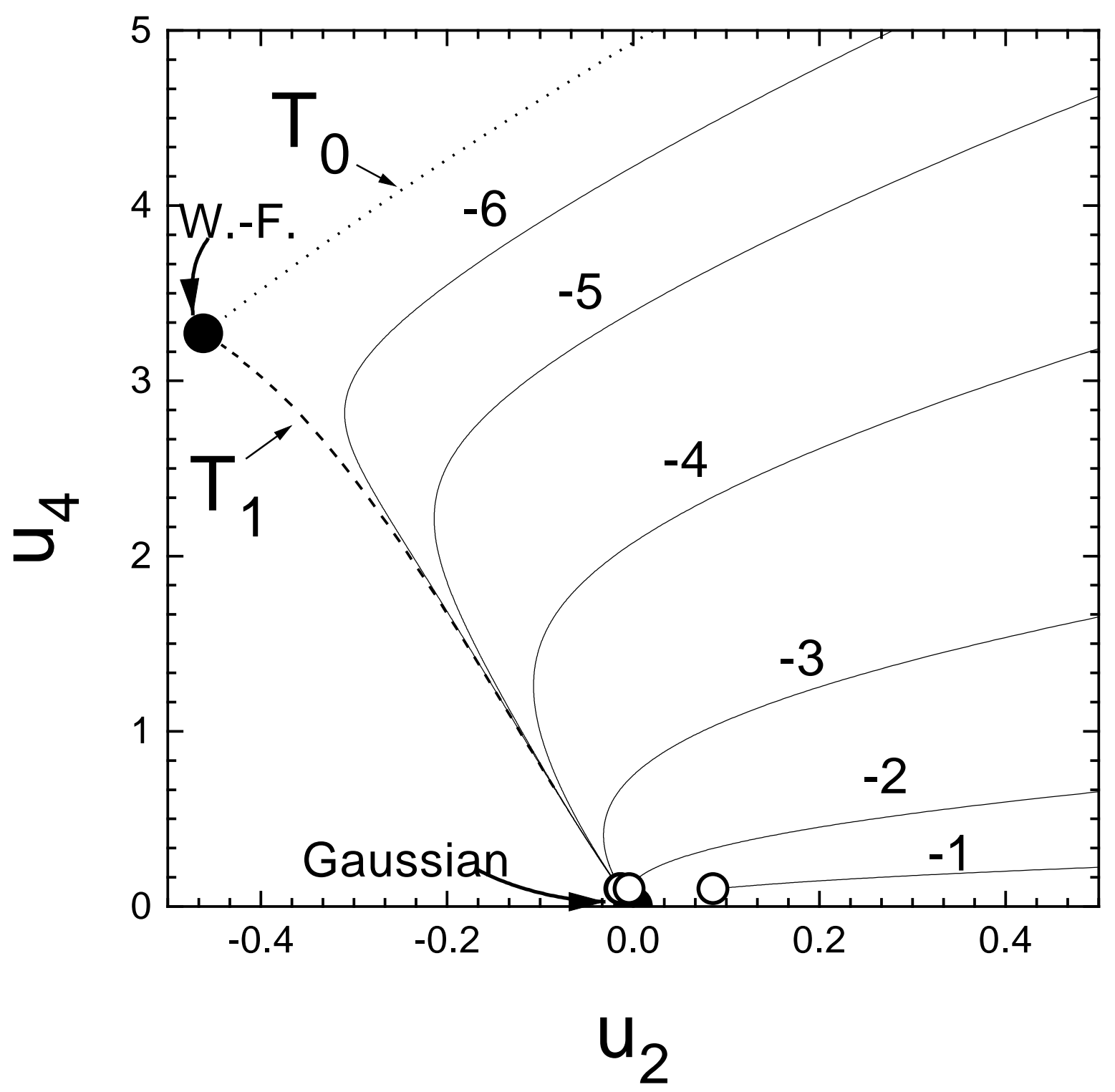




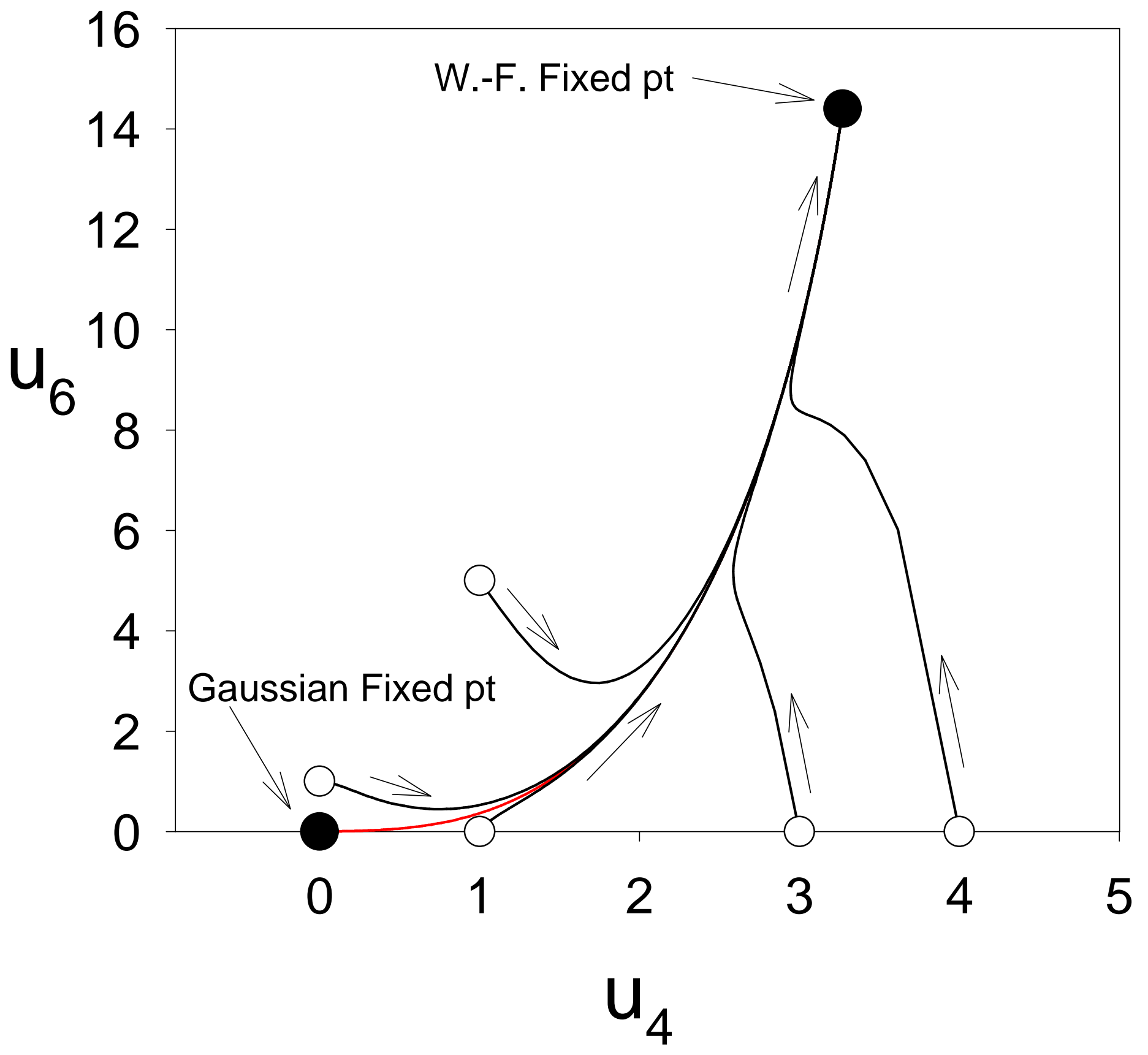



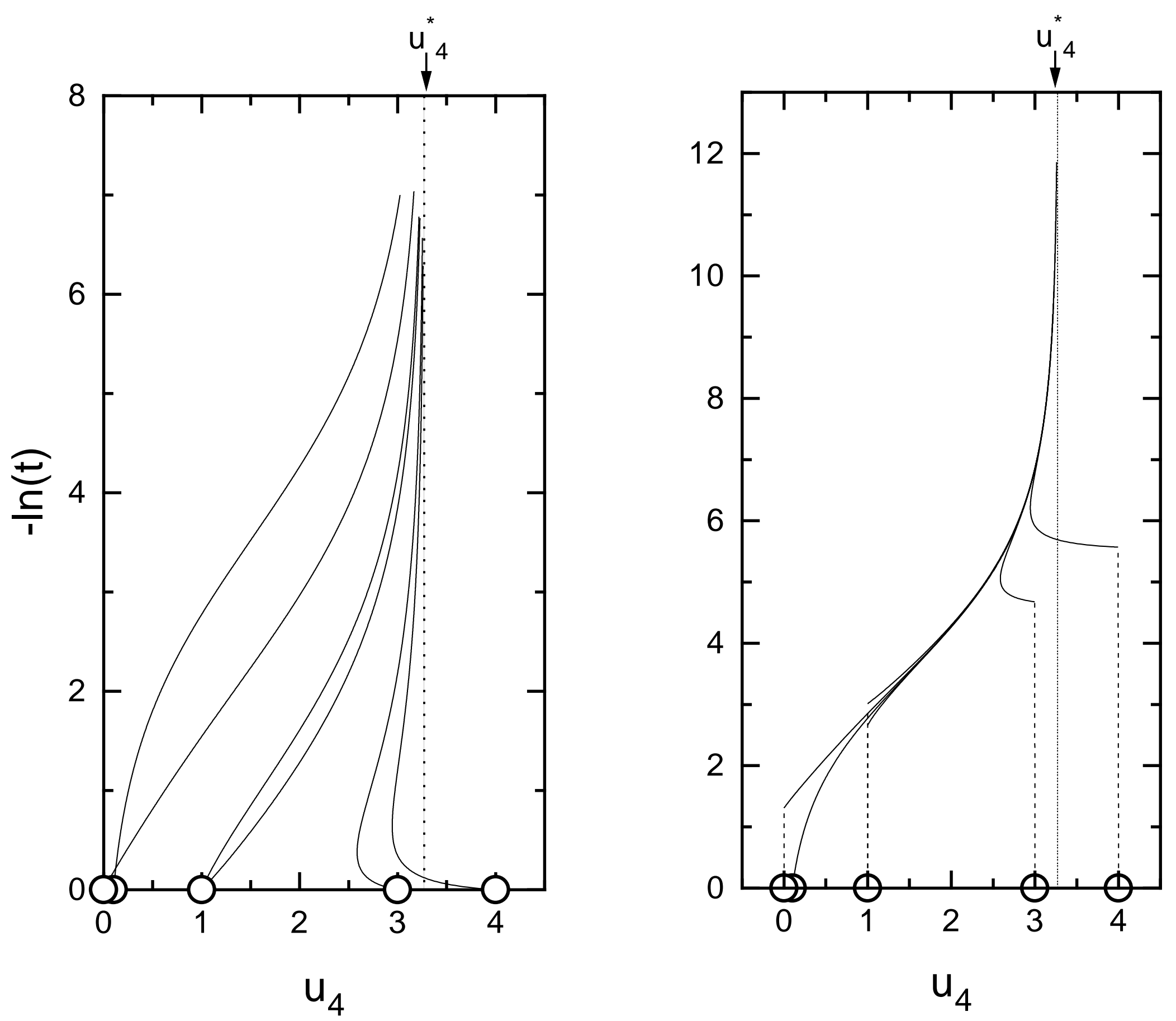


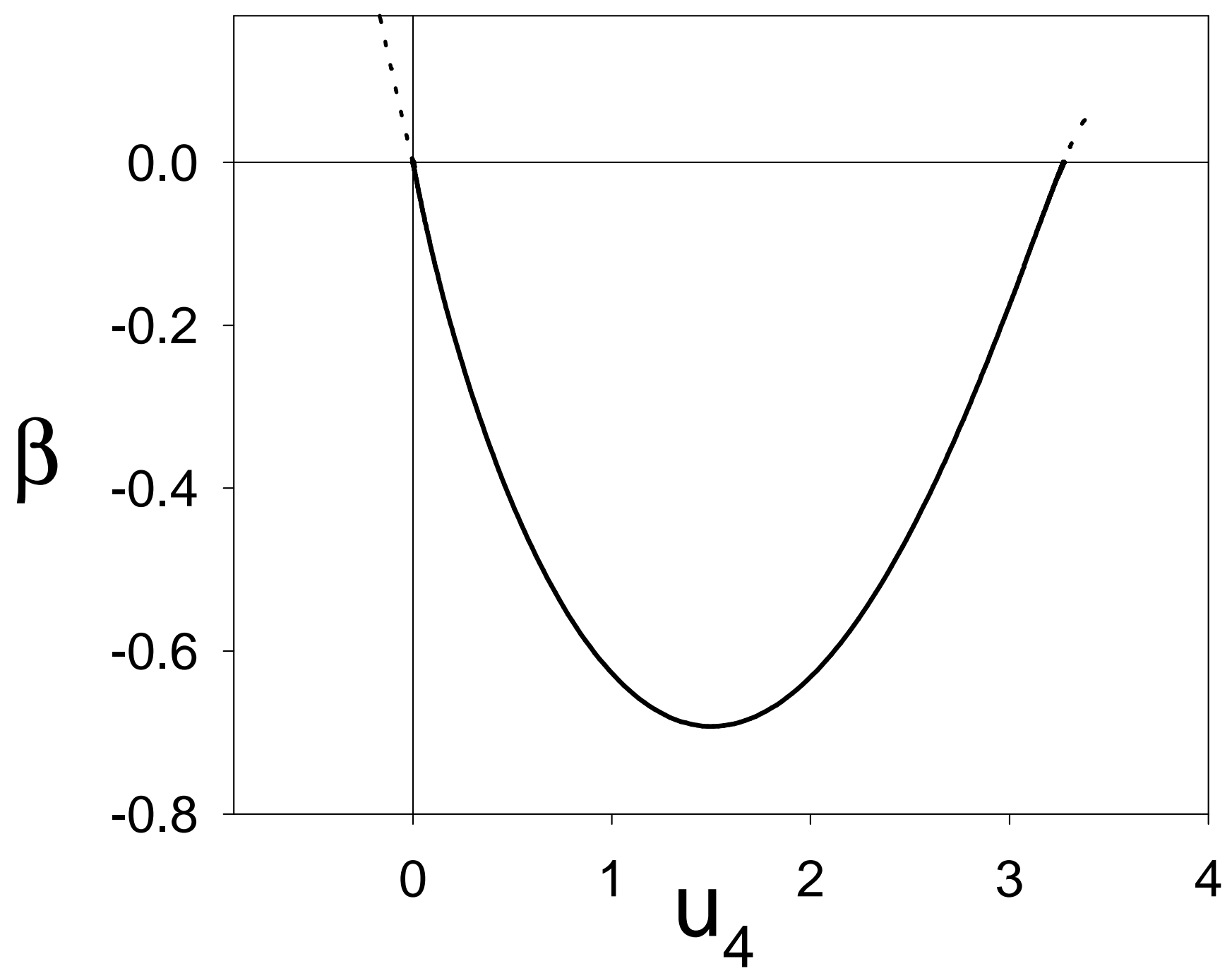




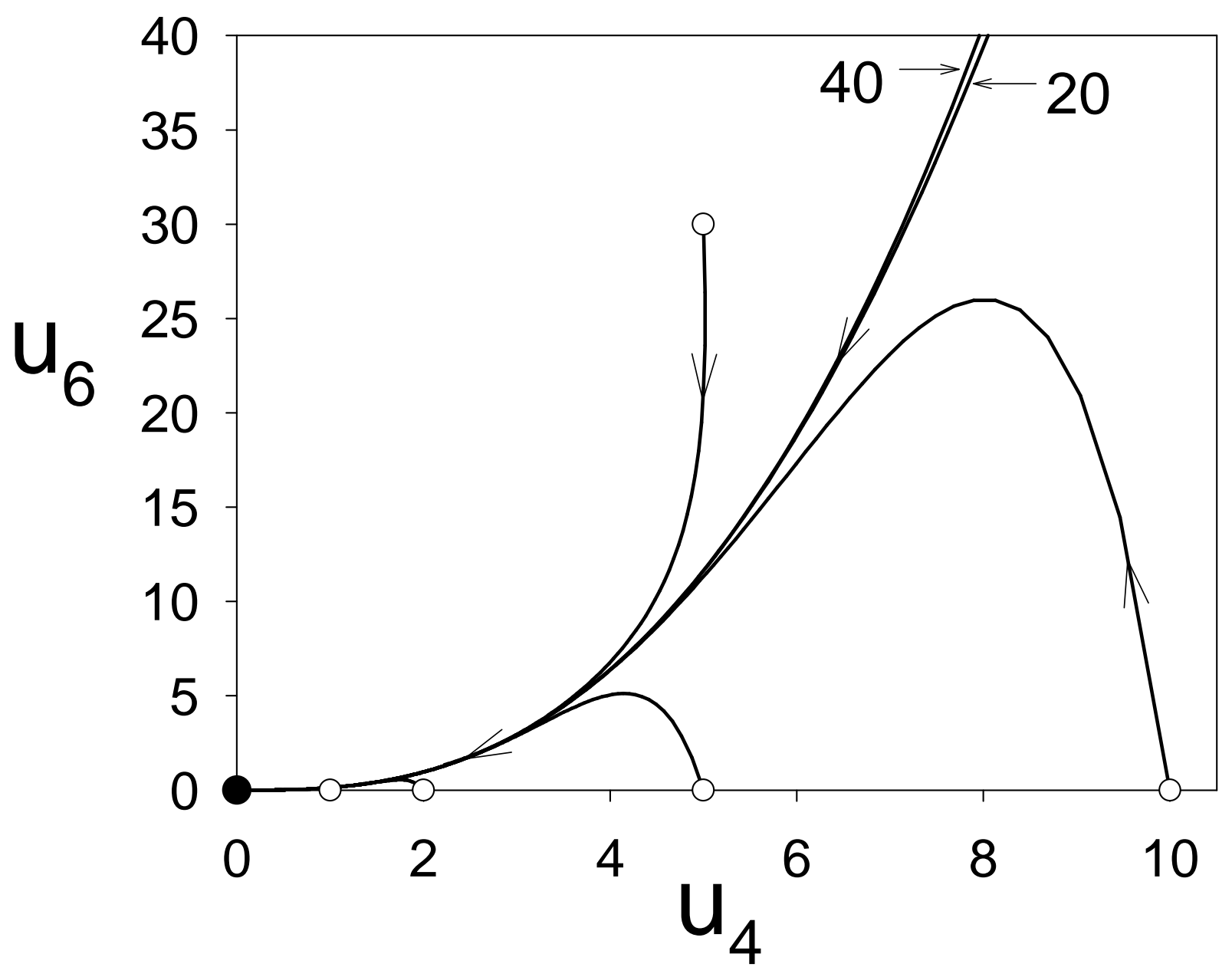

